\title{
TỐI ƯU HOÁ CÁC THÔNG SỐ HOẠT ĐộNG CỦA THIẾT BỊ NHIỆT ĐỊNH HİNH BĂNG PHƯỚNG PHÁP TAGUCHI
}

\author{
NGUYỄN KHOA TRIỀU , CHÂU MINH QUANG, NGUYẼ̃N XUÂN CHIẾN, \\ PHẠM HOÀNG ANH DU'ƠNG, PHẠM MẠNH HÙNG, NGUYẼ̃N TRUNG PHI \\ Khoa Công Nghệ Co Khí, Truờng Đại học Công nghiệp Thành phố Hồ Chí Minh, \\ nguyenkhoatrieu@iuh.edu.vn
}

Tóm tắt. Bài báo này trình bày quá trình tối ưu hóa các thông số hoạt động của một thiết bị nhiệt định hình dùng phương pháp Taguchi và phân tích phương sai ANOVA. Đầu tiên, các tác giả chọn năm thông số quan trọng ảnh hưởng đển sự đồng đều và chất lượng ngoại quan của sản phẩm, bao gồm nhiệt độ cài đặt của nhiệt điện trở, khoảng cách giữa tấm nhựa với nhiệt điện trở, tỷ số kéo của khuôn dương, thời gian gia nhiệt, độ dày của tấm nhựa để thực hiện tối ưu hóa. Sau đó, các thử nghiệm tiền khả thi giúp xác định ba mức giá trị cho mỗi thông số. Và do đó, mảng trực giao $\mathrm{L}_{18}$ là phù hợp cho việc tối ưu hóa này. Dựa trên mảng trực giao này, các tác giả thực hiện 18 thí nghiệm nhiệt định hình để lấy số liệu phân tích. Sau đó, tỷ số $\mathrm{S} / \mathrm{N}$ và phân tích phương sai $\mathrm{ANOVA}$ được dùng để tìm các mức tối ưu của các thông số và tác động của chúng tới độ đồng đều về chiều dày của sản phẩm. Kết quả cho thấy độ dày của tấm nhựa và tỷ số kéo có ảnh hưởng nhiều nhất, lần lượt là $34.90 \%$ và $32.48 \%$. Sau đó, các tác giả thực hiện một thí nghiệm bổ sung để chứng minh tính hiệu quả của phương pháp Taguchi. Kết quả thu được là $0.06 \mathrm{~mm}$, tối ưu hơn 18 kết quả đã thực hiện trước đó. Từ đó, ta có thể kết luận rằng phương pháp Taguchi, phân tích phương sai ANOVA là một công cụ đơn giản nhưng hiệu quả cho tối ưu hóa quá trình một công nghệ sản xuất truyền thống như nhiệt định hình, nơi vốn không được chú ý nghiên cứu khoa học và đầu tư trang thiết bị vật chất cũng như phần mềm hiện đại.

Từ khóa. Nhiệt định hình, Tối ưu hoá, Taguchi, ANOVA, Độ đồng đều, Tỷ số S/N, Minitab.

\section{TAGUCHI OPTIMIZATION OF PROCESS PARAMETERS FOR THICKNESS UNIFORMITY OF A THERMOFORMING PROCESS}

\begin{abstract}
In this article, an investigation for optimization of process parameters for thickness uniformity in a thermoforming process utilizing Taguchi method with analysis of variance (ANOVA) was presented. At first, five parameters, including setting temperature of ceramic heaters, distance between plastic sheet and the heaters, areal draw ratio, heating time, thickness of the plastic sheet were considered in the initial approach towards optimization. As a continuation of previous works, three levels of the processing condition for each parameter were established based on the recommended ranges. Therefore, an $\mathrm{L}_{18}$ Taguchi orthogonal design was selected. As the next step, 18 experiments were carried out by utilizing the combination of process parameters based on this $\mathrm{L}_{18}$ array. Then, the $\mathrm{S} / \mathrm{N}$ ratio and ANOVA were used to find the optimal levels to indicate the impact of the process parameters on the uniformity, showing that thickness of the plastic sheet and areal draw ratio played the most important roles, $34.90 \%$ and $32.48 \%$, corespondingly. A verification test was also performed to demonstrate the effectiveness of Taguchi method, resulting a $0.06 \mathrm{~mm}$ uniformity, better than any previous results. Hence it can be clearly infered from this investigation that Taguchi method is a simple, sufficient tool to optimize such a traditional manufacturing process as thermoforming without using any complicated scientific researches or expensive software solutions.
\end{abstract}

Keywords. Thermoforming, Optimization, Taguchi, ANOVA, Uniformity, S/N ratio, Minitab.

\section{GIỚI THIỆ}

Nhiệt định hình (thermoforming) cùng với ép phun (injection molding) là hai trụ cột trong ngành nhựa, đáp ứng phần lớn nhu cầu về sản phẩm nhựa của con người. Trong khi ép phun cung cấp các sản phẩm công nghiệp và dân dụng thì nhiệt định hình lại phục vụ cho dân dụng và y tế $[1,2]$. Với rất nhiều ưu điểm như 
sản xuất được sản phẩm mỏng có độ đồng đều cao, nhiệt độ thấp, vật liệu khuôn đa dạng, giá thành thiết bị không cao, năng suất cao, số lượng sản xuất lớn, ... nhiệt định hình đóng vai trò ngày càng quan trọng trong ngành nhựa [1].

Cũng như ép phun, nhiệt định hình được phát minh ra cách đây hơn 150 năm, là một kỹ thuật sản xuất nhựa truyền thống. Cũng chính vì thế, trong những nghiên cứu về lĩnh vực sản xuất truyền thống này, để có thể được chấp nhận đăng, các tác giả đã sử dụng những kỹ thuật tối ưu hóa rất phức tạp. Trong [3], các tác giả cố gắng dùng thuật toán để mô hình hóa quá trình nhiệt định hình dùng phương trình Treloar và công thức Rivlin. Nhược điểm của nó là công thức rất phức tạp. Còn trong [4], các tác giả dùng định luật G’Sell và phần mềm $P A M-S T A M P ®$ để tối ưu hóa quá trình nhiệt định hình. Đây là một phần mềm không phổ biến

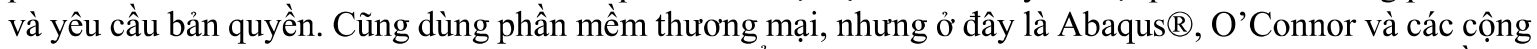
sự [5] dùng các mô hình Ogden và Arruda-Boyce để mô hình hóa quá trình nhiệt định hình dùng phần tử hữu hạn. Sau đó, các tác giả này tiếp tục dùng mô hình đàn nhớt Sweeny để mô phỏng và tối ưu hóa quá trình [6]. Có nhiều tác giả khác cũng sử dụng Abaqus và mô hình Ogden cho quá trình nhiệt định hình [7]. Cũng dùng mô hình Ogden, trong [8], các tác giả kêt hợp với mô hình Mooney-Livlin để cải tiến chất lượng tối ưu hóa. Còn Azdast và các cộng sự [9] thì tiếp tục cải tiến mô hình Mooney-Livlin thành mô hình Mooney-Livlin siêu đàn hồi. Tương tự như vậy, mô hình Lagrangian sửa đổi và phương pháp NewtonRaphson được sử dụng để mô phỏng quá trình nhiệt định hình [10]. Cũng tối ưu hóa quá trình nhiệt định hình, Yang và cộng sự [11] dùng thuật toán di truyền nghịch đảo, một thuật toán khá phức tạp. Còn trong [12], các tác giả dùng thuật toán di truyền kết hợp với phân tích phương sai (ANOVA). Trong đó, quá trình đã được tối ưu hóa, tuy nhiên lợi ích đạt được có tương xứng với độ phức tạp của quá trình tối ưu hay không thì chưa được kiểm chứng. Cũng dùng thuật toán, các tác giả trong [13] dùng phương trình Van der Waals và hàm chuỗi Prony để xây dựng một mô hình gồm 18 tham số để kiểm soát và tối ưu hóa độ dày của sản phẩm nhiệt định hình. Cũng dùng phương trình Van der Waals, nhưng Makradi và các cộng sự từ Pháp [14] kết hợp với mô hình Bergstrom và Boyce dựa trên lý thuyết Doi và Edwards để mô hình hóa quá trình nhiệt định hình. Ngoài nhựa vô định hình [14], các tác giả này cũng ứng dụng mô hình của họ cho nhựa bán tinh thể [15]. Trong khi đó, một nhóm các tác giả đến từ Iran và Nga [16] thì sử dụng mô hình lý thuyết của Leonov kết hợp với các công thức toán từ Cauchy và Lagrangian để mô hình hóa và nghiên cứu tính lưu biến của vật liệu nhựa trong nhiệt định hình. Cũng như các tác giả khác, Abbasi và các cộng sự [17] cũng đưa ra một mô hình toán học với 14 tham số dựa trên các công thức toán học có sẵn. Còn những phương pháp tối ưu hóa truyền thống, nói một cách khác là đơn giản, như Taguchi, thì phải kết hợp với một hay nhiều thuật toán khác. Ví dụ như trong [18], các tác giả kết hợp Taguchi với khái niệm tiện ích (utility concept) hay hàm tiện ích (utility function). Hay trong [19], Yu và các cộng sự phát triển một phương pháp tối ưu hóa quá trình dùng mạng lưới nơ ron Taguchi mờ (fuzzy neural-Taguchi network) dùng thuật toán di truyền, lưới Taguchi lan truyền ngược (back propagation Taguchi network) được dùng để dự đoán mối quan hệ giữa các biến số thiết kế và đặc tính của quy trình, sau đó, thuật toán di truyền được dùng để tối ưu hóa.

Phương pháp Taguchi từ lâu đã được thừa nhận rằng đơn giản nhưng hiệu quả, ngày càng được sử dụng rộng rãi trong cả học thuật lẫn trong công nghiệp, thực tiễn sản xuất [20]. Trong môi trường sản xuất, chế tạo, các thuật toán phức tạp như mô hình Mooney-Livlin siêu đàn hồi, thuật toán di truyền, mạng lưới nơ ron Taguchi mờ, ... rất khó được ứng dụng vì độ phức tạp của chúng. Các nhân viên hoặc công nhân trong xưởng, sau khi phân tích những phương trình 14 hay 18 tham số sẽ không kịp tiến độ giao hàng. Hơn nữa, chất lượng trong sản xuất không phải là hoàn hảo mà là đáp ứng vừa đủ nhu cầu của khách hàng. Do đó, những nghiên cứu khoa học trên rất khó ứng dụng vào thực tế. Vì vậy khoảng cách giữa học thuật và thực tiễn sản xuất ngày càng xa. Nhằm lấp đầy khoảng cách này, một nghiên cứu sử dụng phương pháp Taguchi, phân tích phương sai (ANOVA) đã được thực hiện. Tiếp theo, kết quả tối ưu hóa đã được kiểm tra lại bằng phần mềm thương mại Minitab ${ }^{\circledR}$, sau đó được kiểm chứng bằng một thực nghiệm bồ sung. Kết quả cho thấy độ đồng đều tối ưu trong trường hợp đầu vào tối thiểu đã đạt được. Những lý thuyết và tính toán về Taguchi và $A N O V A$ nhằm để các nhân viên / công nhân hiểu bản chất của quá trình, trong thực tiễn sản xuất, toàn bộ quá trình tối ưu hóa có thể được thực hiện bởi Minitab®, vốn có thể dễ dàng cài đặt ở xưởng. 


\section{VẬT LIỆ VÀ PHƯƠNG PHÁP}

\subsection{Vật liệu polyethylene terephthalate (PET)}

Do những ưu điểm về giá, tính phổ biến, dễ gia công, PET đã được sử dụng làm nguyên liệu thô dạng tấm cho quá trình nhiệt định hình. $\mathrm{PET}$ là nhựa nhiệt dẻo, thuộc vào loại nhựa polyester, được tạo ra bằng cách đa trùng ngưng các monomer $\left(\mathrm{C}_{10} \mathrm{H}_{8} \mathrm{O}_{4}\right)_{\mathrm{n}}$. Uu điểm lớn của PET là có khả năng chịu lực và chịu nhiệt cao, khi được gia nhiệt ở $200^{\circ} \mathrm{C}$ hay làm lạnh ở $-90^{\circ} \mathrm{C}$ thì cấu trúc hóa học của chúng vẫn được giữ nguyên. Các đặc tính chung của nhựa PET nguyên chất được liệt kê trong bảng 1 [21, 22].

Bảng 1: Các thông số của nhựa PET [21, 22].

\begin{tabular}{|c|c|c|c|}
\hline TT & Tính chất & Giá trị & Đơn vị \\
\hline 1 & PET tinh thể & 1,370 & $\mathrm{~g} / \mathrm{cm}^{3}$ \\
\hline 1 & Knô lượng rieng $\quad$ PET vô định hình & 1,445 & $\mathrm{~g} / \mathrm{cm}^{3}$ \\
\hline 2 & Young's modulus (E) & $2,800-3,100$ & $\mathrm{MPa}$ \\
\hline 3 & Độ bền kéo $\left(\sigma_{t}\right)$ & $55-75$ & $\mathrm{MPa}$ \\
\hline 4 & Giới hạn dẻo & $50-150$ & $\%$ \\
\hline 5 & Độ chịu va đập (notch test) & 3.6 & $\mathrm{~kJ} / \mathrm{m}^{2}$ \\
\hline 6 & Nhiệt độ thủy tinh (Glass temperature) & 75 & ${ }^{0} \mathrm{C}$ \\
\hline 7 & Điểm nóng chảy & 260 & ${ }^{0} \mathrm{C}$ \\
\hline 8 & Nhiệt độ hóa mềm Vicat B & 170 & ${ }^{0} \mathrm{C}$ \\
\hline 9 & Hê số dẫn nhiêt & 0.24 & $\mathrm{~W} /(\mathrm{m} \cdot \mathrm{K})$ \\
\hline 10 & Hệ số giãn nở tuyến tính $(\alpha)$ & $7 \times 10^{-5}$ & $1 / \mathrm{K}$ \\
\hline 11 & Nhiệt dung riêng $(\mathrm{C})$ & 1.0 & $\mathrm{~kJ} / \mathrm{kg} \cdot \mathrm{K}$ \\
\hline 12 & Chỉ số hấp thụ nước (ASTM) & 0.16 & - \\
\hline 13 & Chỉ số khúc xạ & 1.5750 & - \\
\hline
\end{tabular}

\subsection{Phương pháp nhiệt định hình}

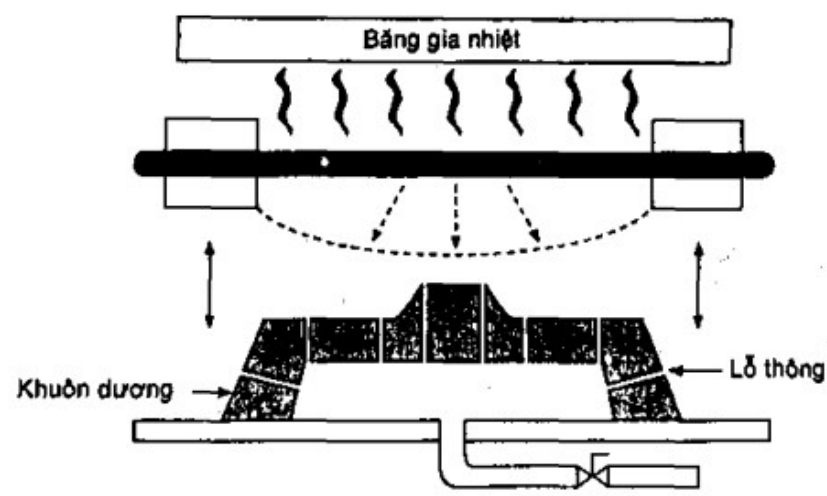

(a) Gia nhişt

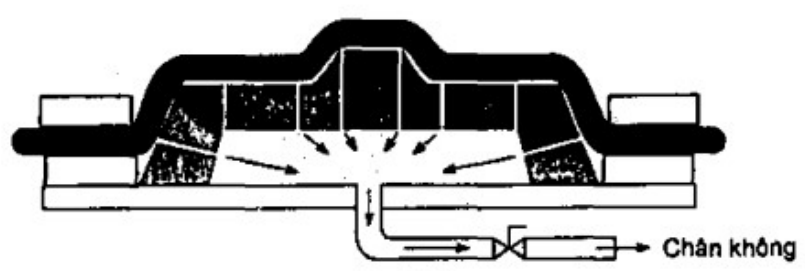

(b) Tao hinh

Hình 1. Tạo hình bằng chân không cơ bản bằng khuôn dương [23]

Như đã trình bày, ép phun và nhiệt định hình là hai trụ cột của ngành nhựa [23]. Trong đó, nhiệt định hình có quy trình và thiết bị đơn giản hơn, hoạt động tại nhiệt độ thấp hơn. Trong các nguyên lý nhiệt định hình 
phổ biến, nguyên lý được lựa chọn là nhiệt định hình dùng tạo hình chân không cơ bản, hình 1 . Tạo hình chân không cơ bản là một phương pháp tạo hình chân không đơn giản nhất mà trong đó chỉ có chân không được sử dụng để ép chất dẻo nóng áp vào bề mặt khuôn [23]. Trong kỹ thuật này, vật liệu được kẹp và được đốt nóng đến điểm lún và sau đó được ép trên khuôn bằng cách di chuyển vật liệu vào bên trên khuôn. Đồng thời, chân không được áp dụng qua lỗ thông ở khuôn, do đó kéo sản phẩm vào bề mặt bên ngoài khuôn. Sản phẩm được làm mát khi tiếp xúc với khuôn tạo thành chi tiết in hình của khuôn. Kỹ thuật tạo hình bằng khuôn dương được chỉ ra trong hình 1.

Lợi thế chính của tạo hình bằng khuôn dương so với tạo hình bằng khuôn âm là độ sâu kéo lớn hơn có thể đạt được mà không có những chỗ mỏng quá mức. Sản phẩm được ép ở trên khuôn với độ mỏng tương đối ít. Bởi vậy, khi tạo hình cuối cùng xảy ra, độ đồng đều về độ dày tốt nhiều hơn so với trường hợp có thể đạt được với một khuôn âm.

\subsection{Phương pháp Taguchi}

Ngày nay, phương pháp Taguchi ngày càng được ứng dụng rộng rãi trong lập quy hoạch thực nghiệm [24]. Phương pháp này góp phần cải thiện năng suất trong quá trình nghiên cứu và phát triển, tạo ra các sản phẩm có chất lượng cao mà giá thành thấp. Nhà nghiên cứu Taguchi đã phát triển phương pháp dựa trên thí nghiệm mảng trực giao (Orthogonal Array) nhằm giảm các tham số của thí nghiệm với sự bố trí thích hợp các điều kiện thuận lợi nhất của các tham số điều khiển được, bảng 2. Các mảng trực giao tạo ra một sự cân bằng giữa các thí nghiệm và tỉ số Signal - to - Noise $(\mathrm{S} / \mathrm{N})$ là hàm logarit của đầu ra mong muốn, thỏa mãn mục đích tối ưu hóa, giúp cho việc phân tích và dự đoán kết quả. Trong phương pháp Taguchi thuật ngữ "Signal" để chỉ giá trị trung bình (giá trị mong muốn) của mục tiêu đầu ra và thuật ngữ "Noise" để chỉ giá trị không mong muốn. Do vậy tỉ số $\mathrm{S} / \mathrm{N}$ được sử dụng để tính sự sai lệch so với giá trị mong muốn [25].

Tỉ số S/N để tối ưu hóa:

Nhỏ hơn-tốt hơn:

$\mathrm{S} / \mathrm{N}=-10 \times \log _{10}\left[(1 / \mathrm{n}) \times \sum\left(\mathrm{y}_{\mathrm{i}}^{2}\right)\right]$

Lớn hơn - tốt hơn:

$\mathrm{S} / \mathrm{N}=-10 \times \log _{10}\left[(1 / \mathrm{n}) \times \sum\left(1 / \mathrm{y}_{\mathrm{i}}^{2}\right)\right]$

Trong đó n là số thí nghiệm, $\mathrm{y}_{\mathrm{i}}$ là kết quả thí nghiệm thứ i.

Bảng 2: Cách chọn mảng trực giao trong phương pháp Taguchi [24, 25].

\begin{tabular}{|c|c|c|c|c|c|c|}
\hline \multirow{2}{*}{ Mảng trực giao } & \multirow{2}{*}{ Số thí nghiệm } & \multirow{2}{*}{$\begin{array}{c}\text { Số thông số } \\
\text { tối đa }\end{array}$} & \multicolumn{5}{|c|}{ Số mức } \\
\cline { 4 - 8 } & & 2 mức & 3 mức & 4 mức & 5 mức \\
\hline L4 & 4 & 3 & 3 & & & \\
\hline L8 & 8 & 7 & 7 & & & \\
\hline L9 & 9 & 4 & & 4 & & \\
\hline L12 & 12 & 11 & 11 & & & \\
\hline L16 & 16 & 15 & 15 & & & \\
\hline L'16 & 16 & 5 & & & 5 & \\
\hline L18 & 18 & 8 & 1 & 7 & & \\
\hline L25 & 25 & 6 & & & & 6 \\
\hline L27 & 27 & 13 & & 13 & & \\
\hline
\end{tabular}


BĂNG PHƯƠNG PHÁP TAGUCHI

\begin{tabular}{|c|c|c|c|c|c|c|}
\hline L32 & 32 & 31 & 31 & & & \\
\hline L'32 & 32 & 10 & 1 & & 9 & \\
\hline L36 & 36 & 23 & 11 & 12 & & \\
\hline L'36 & 36 & 16 & 3 & 13 & & \\
\hline L50 & 50 & 12 & 1 & & & 11 \\
\hline L54 & 54 & 26 & 1 & 25 & & \\
\hline L64 & 64 & 63 & 63 & & & \\
\hline L'64 & 64 & 21 & & & 21 & \\
\hline L81 & 81 & 40 & & 40 & & \\
\hline
\end{tabular}

Thiết kế của các thí nghiệm sử dụng phương pháp Taguchi là hiệu quả hơn so với phương pháp thống kê khác. Bằng cách chọn đúng mức độ phù hợp của các biến độc lập khác nhau, số lượng các thí nghiệm được giảm đáng kể. Cùng lúc đó, không mất mát bất kỳ thông tin nào do giảm số thí nghiệm. Mục tiêu của Taguchi đã giảm thiểu sự thay đổi xung quanh mục tiêu và nâng cao chất lượng. Việc sử dụng và học tập kỹ thuật này cho phép các kỹ sư, nhà khoa học và nghiên cứu cần ít thời gian cho quy hoạch thực nghiệm và phân tích kết quả hơn.Với những cải tiến trong công nghệ máy tính, nó đang trở thành dễ dàng hơn để sử dụng phương pháp Taguchi trong các ứng dụng. Phổ biến nhất là phân tích kỹ thuật dùng các phần mềm máy tính [18].

\subsection{Lưu đồ quy trình tối ưu hóa dùng phương pháp Taguchi}

Lưu đồ quy trình tối ưu hóa được thực hiện như minh họa trong hình 2. Cũng cần lưu ý rằng, ngoài đặc tính chất lượng được dùng để đánh giá quá trình tối ưu hóa là độ đồng đều về độ dày của sản phẩm nhiệt định hình, thêm hai điều kiện ràng buộc được sử dụng. Đó là khả năng tạo hình, sao chép lại hình dạng của khuôn và màu sắc của sản phẩm. Hai điều kiện ràng buộc này được kiểm tra ngoại quan, tương tự như trong môi trường sản xuất.

\section{KẾT QUẢ VÀ THẢO LUẬn}

\subsection{Lựa chọn các tham số quá trình và mảng trực giao}

Thiết bị nhiệt định hình dùng cho nghiên cứu này được trình bày trong hình 4 .

Trong quá trình nhiệt định hình, một tấm vật liệu nhựa nhiệt dẻo (3) được kẹp vào khung kẹp gồm tấm kẹp trên (2) và tấm kẹp dưới (9). Tấm nhựa được nung nóng đến trên nhiệt độ chuyển pha thủy tinh nhờ nhiệt điện trở để nó trở thành nên mềm dẻo. Sau đó, tấm nhựa này được di chuyển đến tiếp xúc với khuôn dương (16) bằng cách thủ công nhờ các thanh trượt (11) được gắn trên khung máy (1). Tiếp theo, tấm nhựa vẫn còn ở trạng thái dẻo được kéo dãn để có thể sao chép lại hình dạng của khuôn bằng cách dùng chênh lệch áp suất nhờ buồng hút (4).

Trong quá trình tạo hình, các tấm nhựa thay đổi chiều dày, điều này cần thiết để tối ưu hóa quy trình trước khi tẩm nhựa nguội. Các tham số quá trình sau đây có thể ảnh hưởng đến chất lượng của quá trình nhiệt định hình [18]:

(1) Các thông số liên quan đến nhiệt độ gồm nhiệt độ gia nhiệt và thời gian gia nhiệt;

(2) Các thông số liên quan đến vật liệu gồm loại vật liệu, độ dày tấm;

(3) Các thông số liên quan đến áp suất gồm áp suất chân không, áp suất dương hỗ trợ;

(4) Các thông số liên quan đến khuôn gồm loại khuôn, tỷ số kéo, chày ép, tốc độ di chuyển và khoảng cách di chuyển.

Qua các nghiên cứu trước đây [26], năm tham số quá trình sau đây đã được xác định là có khả năng quan trọng trong việc ảnh hưởng đến chất lượng đặc điểm của các sản phẩm nhiệt định hình đang nghiên cứu. Gồm nhiệt độ của nhiệt điện trở $\left({ }^{\circ} \mathrm{C}\right)$, khoảng cách gia nhiệt $(\mathrm{mm})$, thời gian gia nhiệt $(\mathrm{s})$, tỷ số kéo $\mathrm{R}_{\mathrm{a}}(-)$, độ dày của tấm vật liệu (mm). 


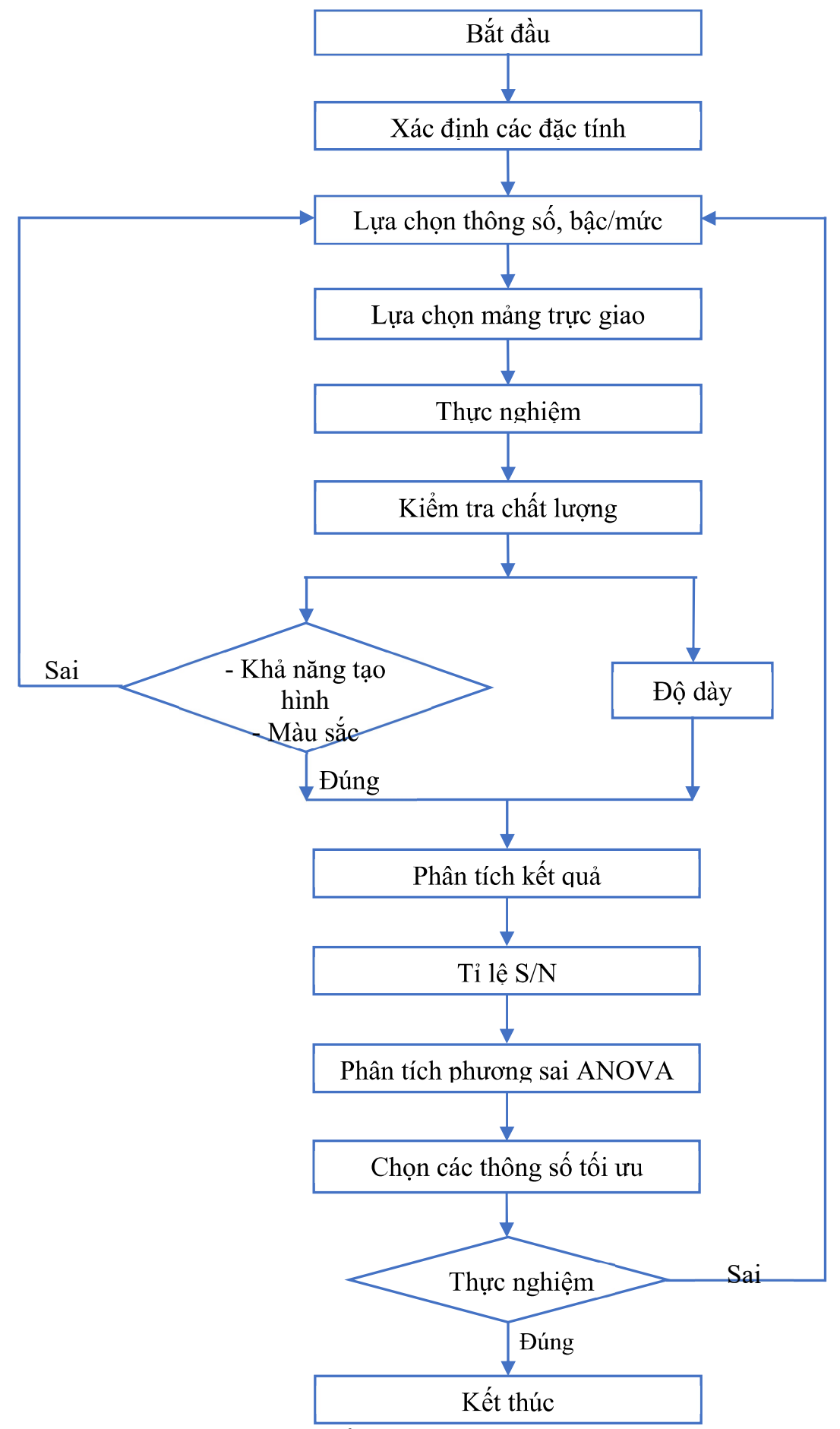

Hình 2. Quy trình tối ưu hóa quá trình nhiệt định hình.

Để chọn phạm vi tham số để đánh giá, một vài thử nghiệm thử nghiệm đã được hoàn thành trước tiên để xác định phạm vi của các giá trị tham số mà các sản phẩm có thể được tạo hình thành công. Các thử nghiệm này được thực hiện dựa trên kinh nghiệm của các chuyên gia, tham khảo thông số của các thiết bị hiện có trên thị trường và phương pháp thực nghiệm thử và sai. Ở giai đoạn này, tấm nhựa PET dày $0.25 \mathrm{~mm}$ được 


\section{TỐI UUU HOÁ CÁC THÔNG SỐ HOẠT ĐỘNG CỦA THIÉT BỊ NHIẸTT ĐỊNH HİNH \\ BẰNG PHƯƠNG PHÁP TAGUCHI}

sử dụng. Đến khi sản phẩm đạt yêu cầu, in hình khuôn hoàn toàn, hình 3 , thì dừng lại và chuyển sang bước nghiên cứu ảnh hưởng và tối ưu hóa thông số hoạt động. Các thông số này được trình bày trong bảng 3 .
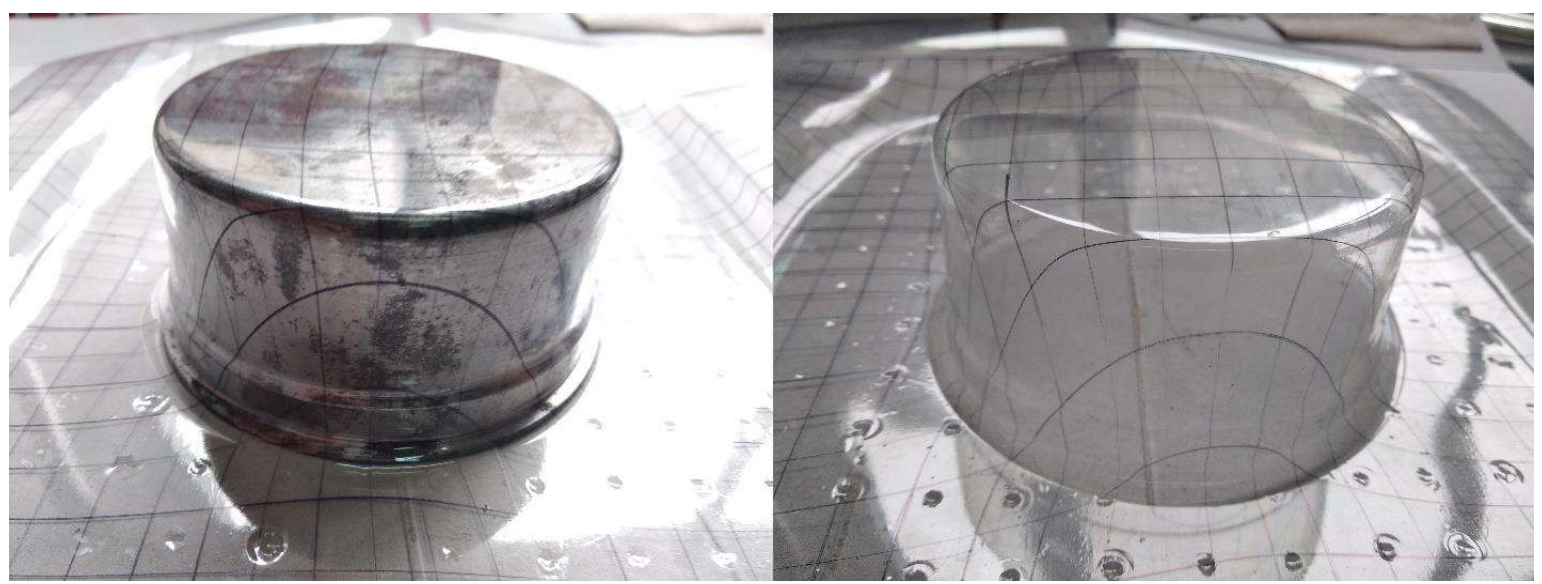

Hình 3. Sản phẩm được chia lưới.

Bảng 3: Các thông số vận hành thiết bị.

\begin{tabular}{|c|l|c|c|}
\hline TT & \multicolumn{1}{|c|}{ Thông số } & Giá trị & Đơn vị \\
\hline 1 & Nhiệt độ & 300 & ${ }^{0} \mathrm{C}$ \\
2 & Thời gian gia nhiệt & 27 & $\mathrm{~s}$ \\
3 & Khoảng cách giữa tấm nhựa với bộ gia nhiệt & 40 & $\mathrm{~mm}$ \\
\hline
\end{tabular}

Bảng 4 liệt kê các yếu tố và mức độ yếu tố được chọn trong thí nghiệm chính.

Bảng 4: Các thông số vận hành thiết bị.

\begin{tabular}{cclcccc}
\hline TT & Ký hiệu & \multicolumn{1}{c}{ Thông số } & Mức 1 & Mức 2 & Mức 3 & Đơn vị \\
\hline 1 & $\mathrm{~A}$ & Nhiệt độ & 290 & 300 & 310 & ${ }^{0} \mathrm{C}$ \\
2 & $\mathrm{~B}$ & Khoảng cách & 38 & 40 & 42 & $\mathrm{~mm}$ \\
3 & $\mathrm{C}$ & Thời gian gia nhiệt & 26 & 27 & 28 & $\mathrm{~s}$ \\
4 & $\mathrm{D}$ & Tỷ số kéo & 1.5 & 2 & 2.5 & - \\
5 & $\mathrm{E}$ & Độ dày & 0.2 & 0.25 & 0.3 & $\mathrm{~mm}$ \\
\hline
\end{tabular}


(a)

(b)
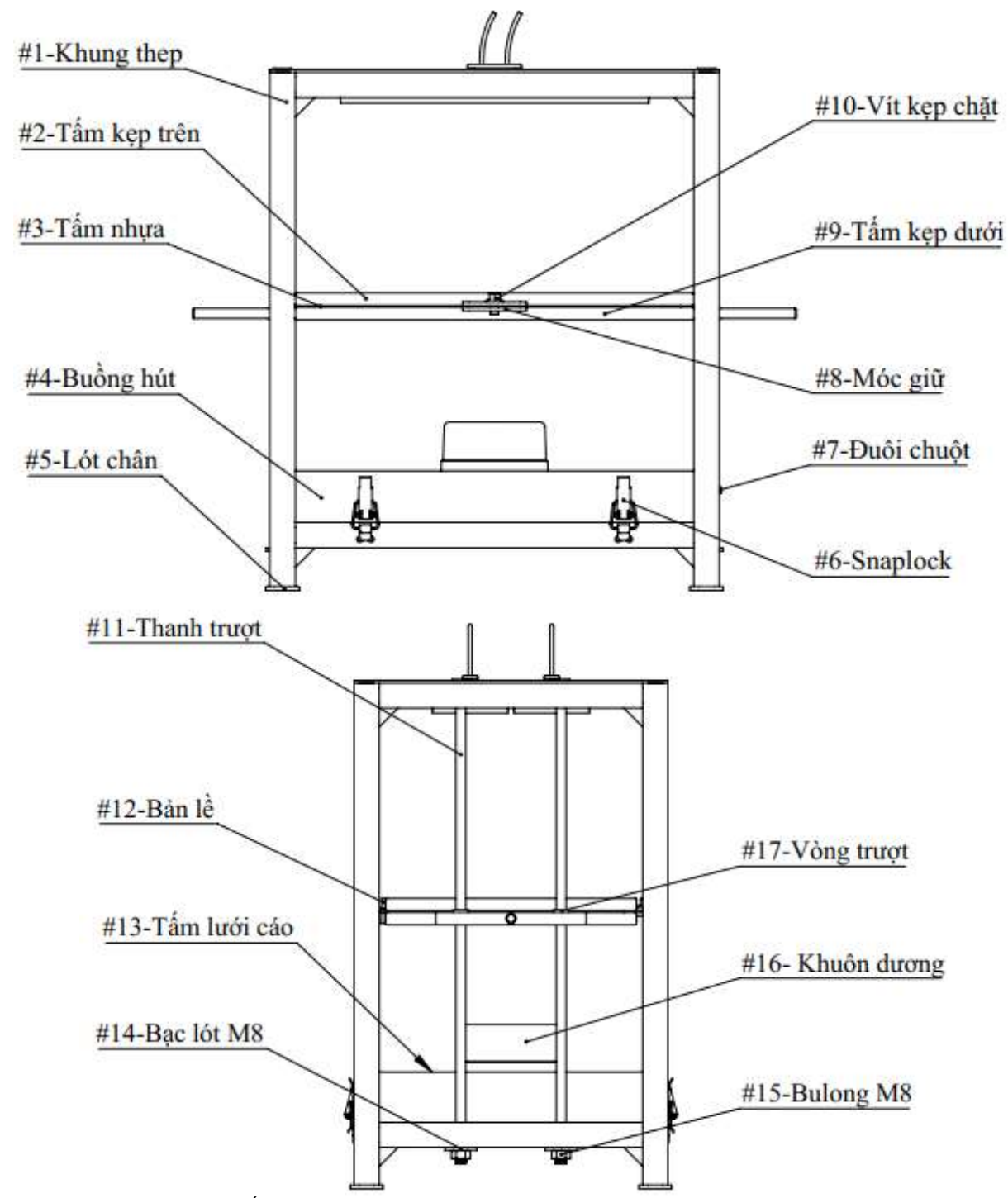

Hình 4. Thiết bị nhiệt định hình [26]: (a) Chính diện, (b) Cạnh bên

Việc xây dựng mảng trực giao phụ thuộc vào số yếu tố và số mức của từng yếu tố [27]. Trong trường hợp này, với 5 yếu tố, mỗi yếu tố 3 mức, mảng trực giao có cấu trúc $\mathrm{L}_{18}$ như trong bảng 5 .

Hình 5 trình bày mô hình 3D của thiết bị nhiệt định hình được xây dựng bằng phần mềm SolidWorks ${ }^{\circledR}$. 


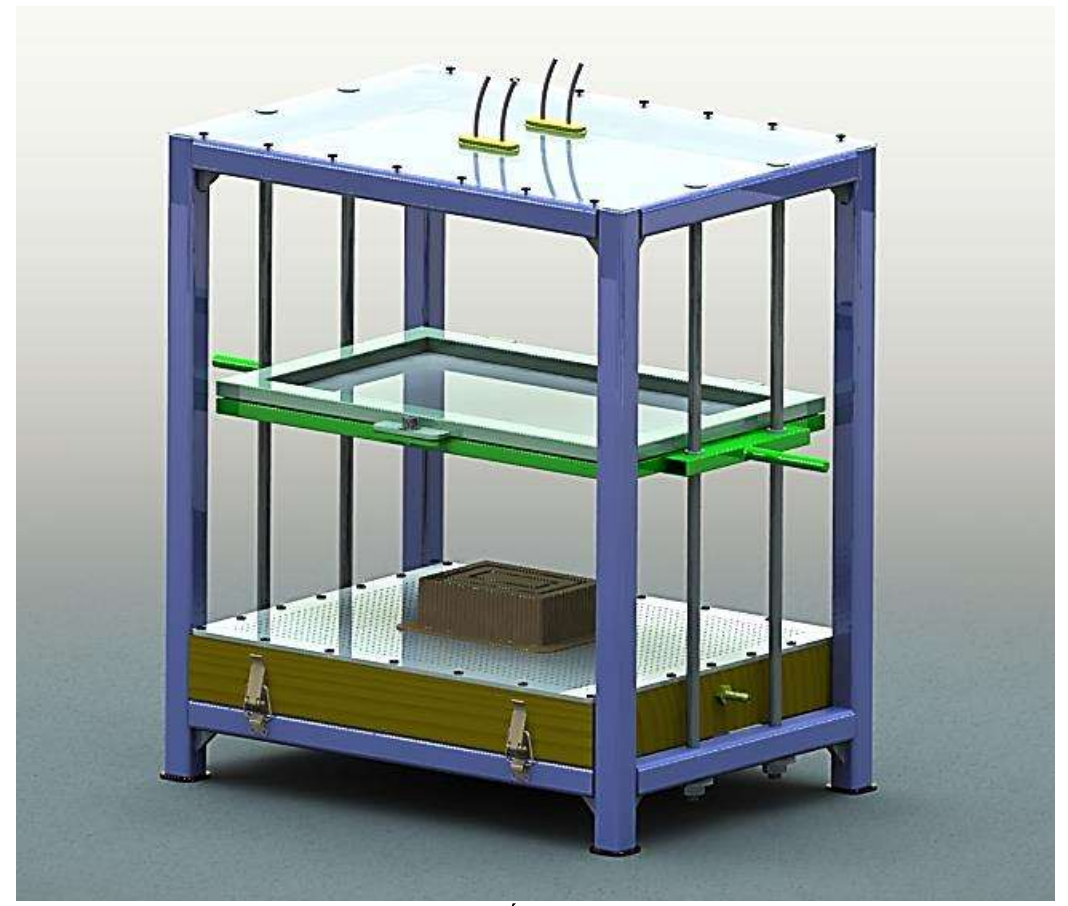

Hình 5. Minh họa thiết bị nhiệt định hình [26].

Bảng 5: Cấu trúc mảng trực giao $\mathrm{L}_{18}$.

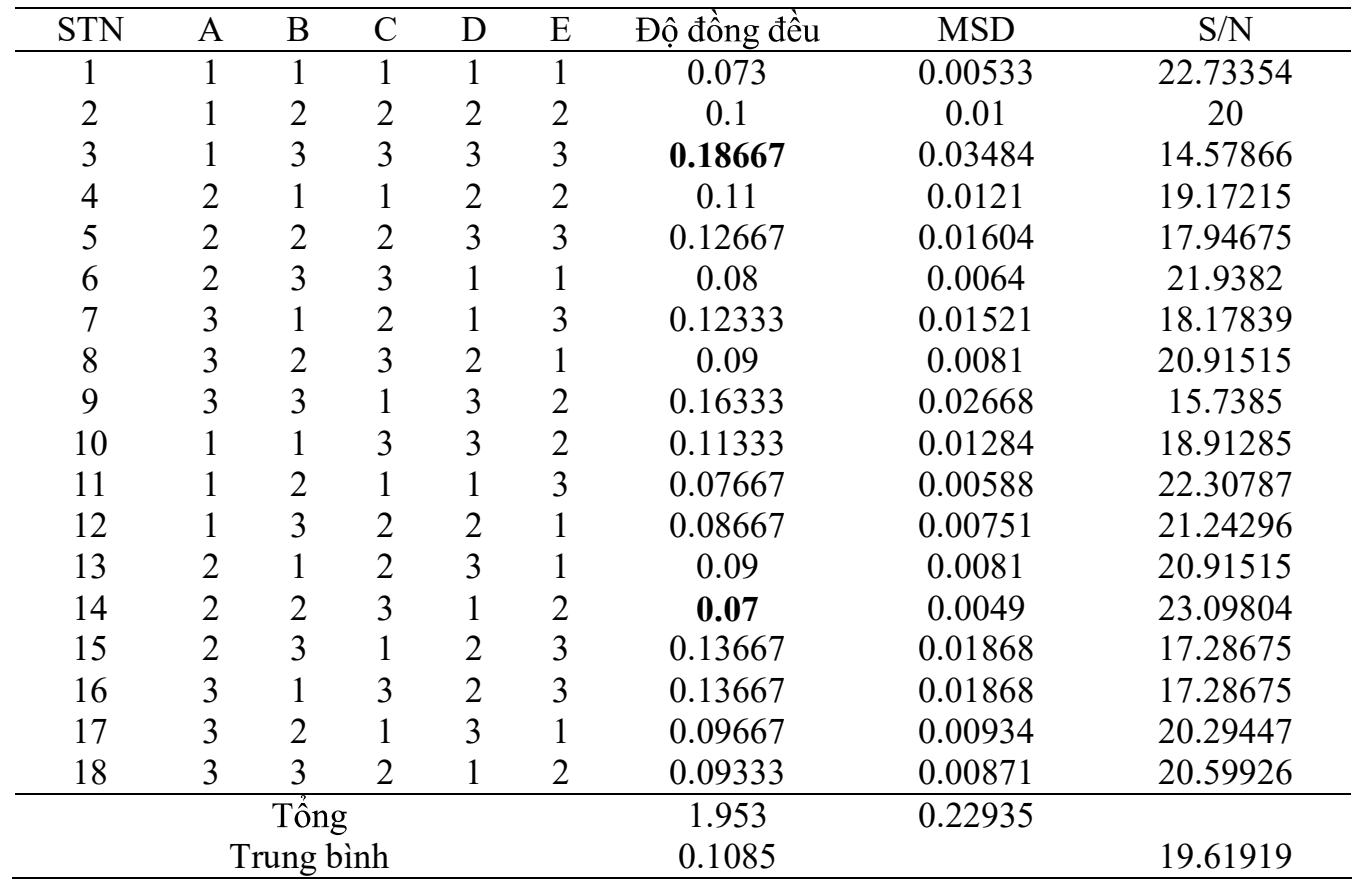

Trong tính toán thống kê, độ lệch toàn phương trung bình, viết tắt MSD (Mean squared diviation) của một phép ước lượng là trung bình của bình phương các độ lệch (sai số), tức là sự khác biệt giữa các ước lượng và những gì được đánh giá. MSD là một hàm rủi ro, tương ứng với giá trị kỳ vọng của sự mất mát độ lệch bình phương hoặc mất mát bậc hai. Sự khác biệt xảy ra do ngẫu nhiên, hoặc vì các ước lượng không tính đến thông tin có thể cho ra một ước tính chính xác hơn [27]. 


\subsection{Kết quả $\mathrm{S} / \mathrm{N}$}

Để tính toán độ nhiễu, hàm đưa ra để tính toán là $\mathrm{S} / \mathrm{N}$ (signal/noise). Mục đích của bài báo là tối ưu độ đồng đều, do đó nguyên lý "nhỏ hơn-tốt hơn" được chọn và được biểu diễn bằng phương trình (1). Kết quả phân tích tỷ số $\mathrm{S} / \mathrm{N}$ được biểu diễn trong hình 6 , cho thấy độ đồng đều của sản phẩm nhiệt định hình tăng khi tỷ số kéo càng nhỏ và chiều dày tấm vật liệu càng nhỏ. Đồng thời, các thông số khác nên được duy trì ở giá trị trung tâm.

Bảng 6: Kết quả tỷ số $\mathrm{S} / \mathrm{N}$ cho mỗi thông số cài đặt.

\begin{tabular}{|c|c|c|c|c|c|c|c|}
\hline \multicolumn{2}{|l|}{ Yếu tố } & A & B & $\mathrm{C}$ & $\mathrm{D}$ & $\mathrm{E}$ & Tổng cộng \\
\hline \multirow{3}{*}{$\begin{array}{l}\text { Tổng tỷ } \\
\text { lệ SN }\end{array}$} & Mức 1 & 19.96265 & 19.53314 & 19.58888 & 21.47588 & 21.33991 & \multirow{3}{*}{58.85757} \\
\hline & Mức 2 & 20.05951 & 20.76038 & 19.81375 & 19.31729 & 19.58680 & \\
\hline & Mức 3 & 18.83542 & 18.56406 & 19.45494 & 18.06440 & 17.93086 & \\
\hline \multicolumn{2}{|c|}{ Độ chênh lệch } & 1.22409 & 2.19632 & 0.35881 & 3.41149 & 3.40905 & 10.59976 \\
\hline \multicolumn{2}{|c|}{ Phần trăm \% } & 11.55 & 20.72 & 3.39 & 32.18 & 32.16 & 100 \\
\hline
\end{tabular}

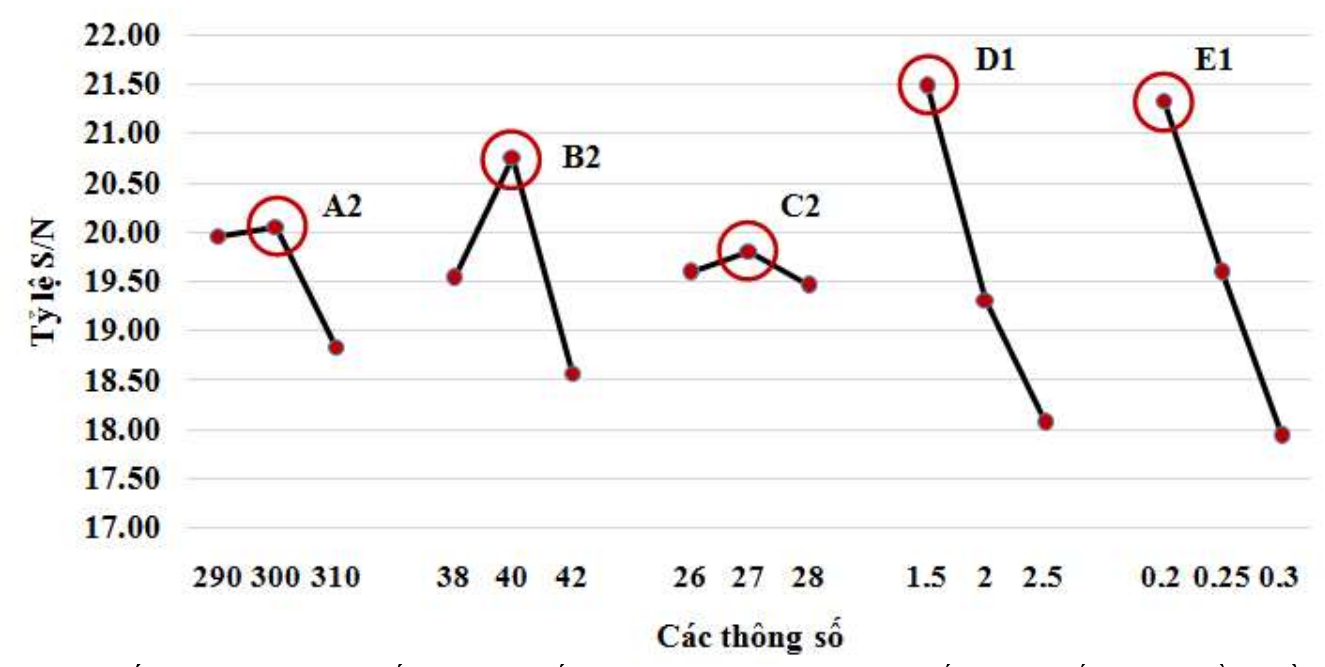

Hình 6. Kết quả phân tích tỷ số $\mathrm{S} / \mathrm{N}$ cho thấy ảnh hưởng của các thông số cài đặt đối với độ đồng đều.

\subsection{Kết quả phân tích ANOVA}

Để tiếp tục nghiên cứu mức độ ảnh hưởng của các thông số cài đặt có liên quan, phương pháp ANOVA được thực hiện như trong bảng 7 . Phù hợp với phân tích tỷ số $\mathrm{S} / \mathrm{N}$, tỷ số kéo càng nhỏ và chiều dày tấm vật liệu có ảnh hưởng lớn tới việc giảm độ chênh lệch về độ dày. Mặc dù có xu hướng tương tự giữa tỷ số $\mathrm{S} / \mathrm{N}$ và phân tích ANOVA, có sự khác biệt nhỏ về độ lớn của các thông số cài đặt. Phương pháp ANOVA chỉ cho thấy hai thông số gồm tỷ số kéo và chiều dày tấm vật liệu, vì có giá trị $\mathrm{F}$ lớn hơn $\mathrm{F}_{(0,01,2,7)}$, là có ý nghĩa thống kê. Chúng đóng góp lần lượt là $32.48 \%$ và $34.90 \%$ vào sự biến thiên của đặc tính chất lượng đang khảo sát. 
BẰNG PHUOONG PHÁP TAGUCHI

Bảng 7: Bảng kết quả ANOVA.

\begin{tabular}{llllllll}
\hline & $\mathrm{S}$ & $\mathrm{f}$ & $\mathrm{V}$ & $\mathrm{F}$ & $\mathrm{F}_{(0.01,2,7)}$ & $\mathrm{P} \%$ & Xếp hạng \\
$\mathrm{A}$ & 0.00072878 & 2 & 0.000364389 & 1.43315 & 9.54658 & 4.18 & 4 \\
$\mathrm{~B}$ & 0.00290915 & 2 & 0.001454574 & 5.72089 & 9.54658 & 16.67 & 3 \\
$\mathrm{C}$ & 0.0002747 & 2 & 0.000137352 & 0.54021 & 9.54658 & 1.57 & 5 \\
$\mathrm{D}$ & 0.00566804 & 2 & 0.002834019 & $\mathbf{1 1 . 1 4 6 2 9}$ & 9.54658 & $\mathbf{3 2 . 4 8}$ & 2 \\
E & 0.00609026 & 2 & 0.00304513 & $\mathbf{1 1 . 9 7 6 6 0}$ & 9.54658 & $\mathbf{3 4 . 9 0}$ & 1 \\
Sai số & 0.0017798 & 7 & 0.000254257 & & & & \\
Tổng cộng & 0.01745072 & 17 & & & & & \\
\hline
\end{tabular}

\subsection{Kiểm tra xác thực}

Từ phân tích tỷ số $\mathrm{S} / \mathrm{N}$, ta có thể xác định rằng độ đồng đều về độ dày tối thiểu có thể đạt được từ điều kiện thông số quá trình A2-B2-C2-D1-E1 như được chỉ ra trong hình 6 . Kết quả này được kiểm tra xác thực bằng phần mềm Minitab ${ }^{\circledR}$. Kết quả xác thực (hình 7 ) cho thấy sự nhất quán với kết quả tính thủ công bằng Excel ${ }^{\circledR}$. Điều này cho thấy tính chính xác của quá trình vận dụng công thức. Thực tiễn cho thấy, các kỹ sư trong các phân xưởng sản xuất chỉ vận dụng phần mềm, chẳng hạn như Minitab®, để tối thiểu hóa thời gian quy hoạch thực nghiệm và phân tích kết quả.

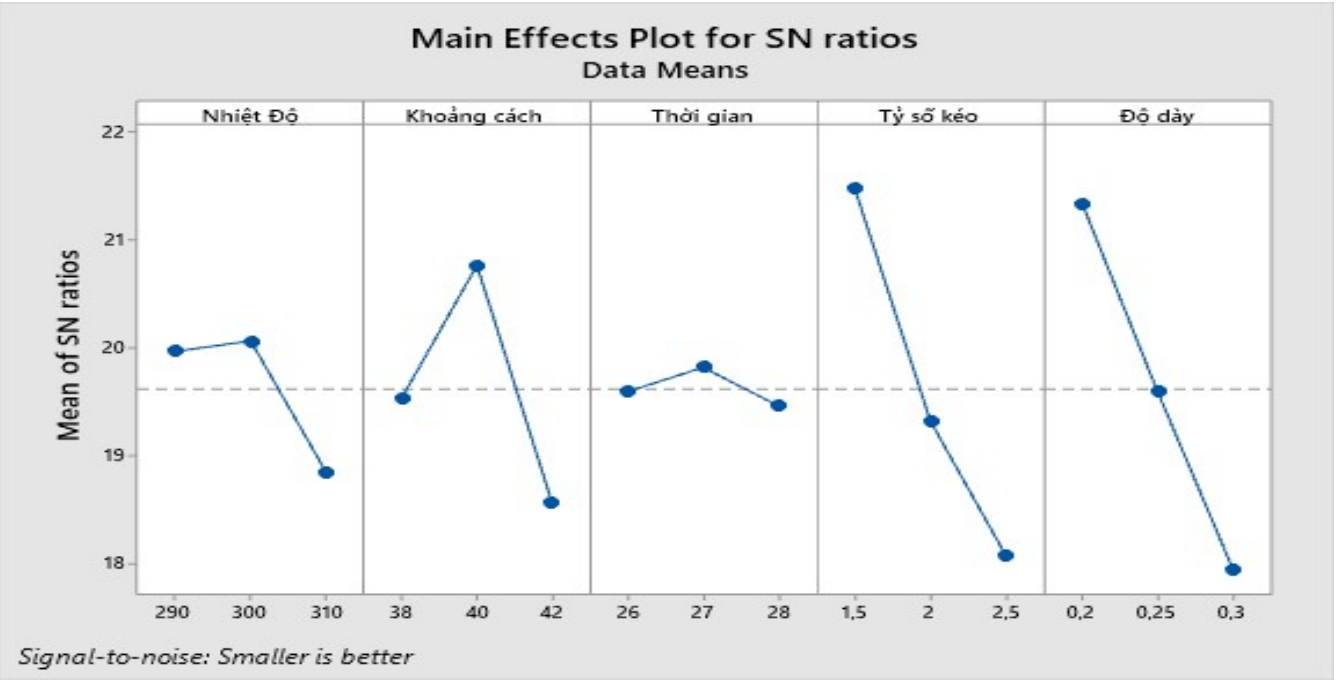

Hình 7. Kết quả thực hiện phương pháp Taguchi bằng phần mềm Minitab®.

Thêm một thực nghiệm nữa được thực hiện, dùng những điều kiện này để xác nhận, hình 8 . Kết quả cho thấy rằng độ đồng đều về độ dày của sản phẩm là $0.06 \mathrm{~mm}$, vốn được định nghĩa là hiệu số giữa độ dày cực đại và cực tiểu trong vùng tạo hình, hình 9 . Giá trị này thấp hơn giá trị độ đồng đều của tất cả các trường hợp được xem xét trong mảng trực giao được liệt kê trong bảng 5 .

Từ thử nghiệm xác nhận này, ta có thể kết luận được rằng phương pháp Taguchi có thể tạo ra một sự kết hợp các thông số quá trình tốt hơn để có thể giảm thiểu độ không đồng đều về độ dày của sản phẩm. 


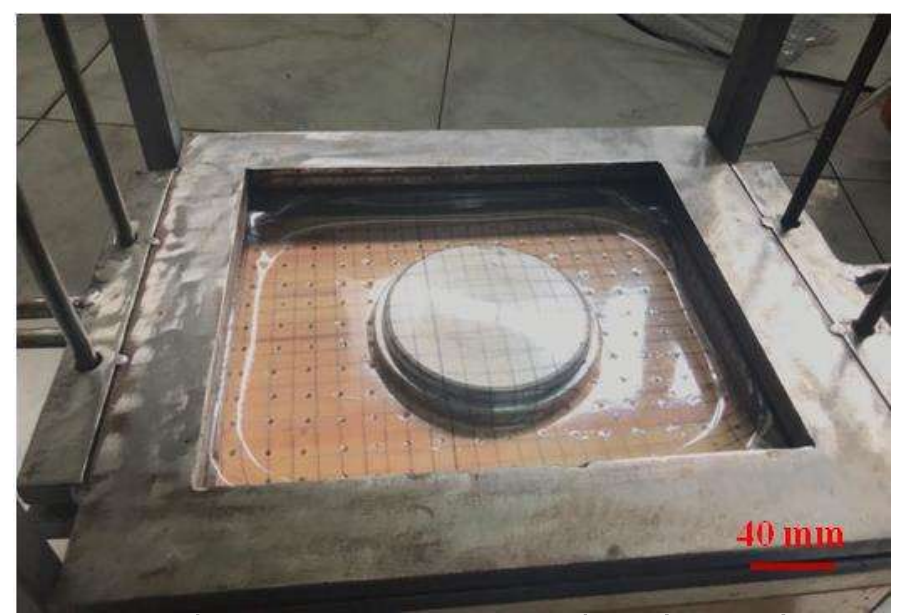

Hình 8. Kết quả thực nghiệm xác thực bằng điều kiện tối ưu

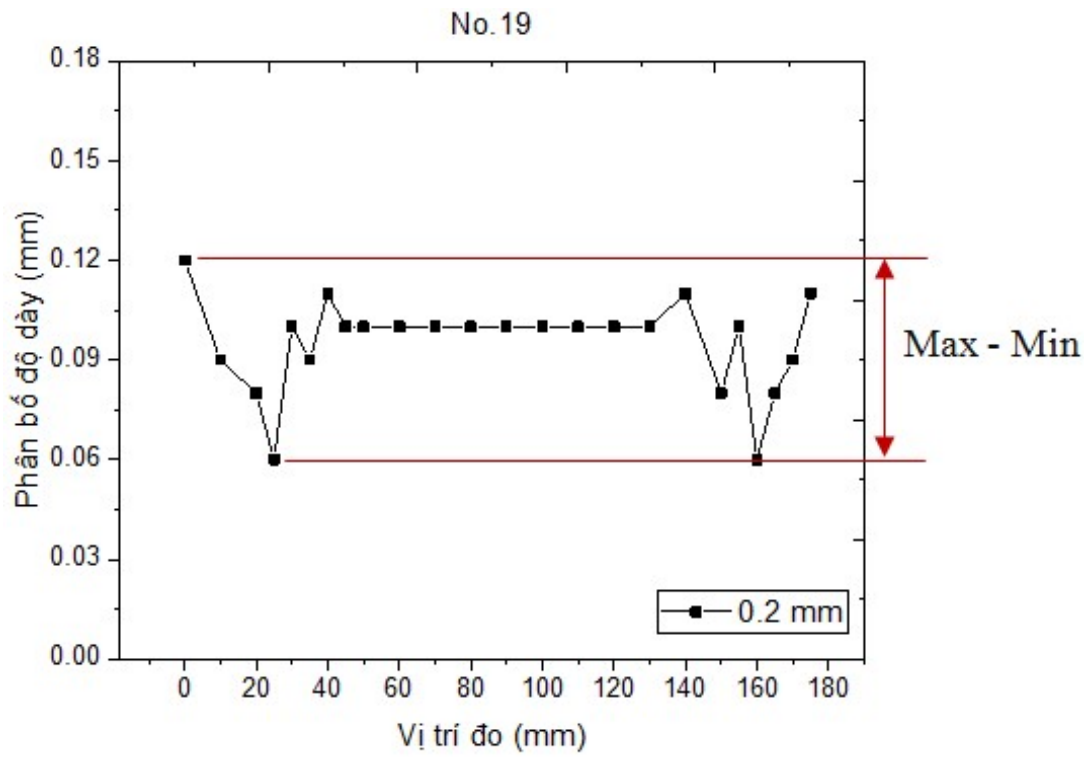

Hình 9. Phân tích kết quả thực nghiệm xác thực bằng điều kiện tối ưu

\section{KẾT LUẤN VÀ ĐỀ NGHI!}

Trong nghiên cứu này, phương pháp Taguchi được sử dụng để nghiên cứu ảnh hưởng của các thông số quá trình lên độ đồng đều về độ dày của các sản phẩm nhiệt định hình. Kỹ thuật tối ưu hóa đơn giản được sử dụng trong nghiên cứu này về cơ bản bao gồm kỹ thuật mảng trực giao của phương pháp Taguchi được sử dụng kết hợp với phân tích phương sai ANOVA. Trong quá trình nhiệt định hình cơ bản dùng khuôn dương, năm thông số quá trình được xem xét. Từ phân tích tỷ số $\mathrm{S} / \mathrm{N}$ và $\mathrm{ANOVA}$ dựa trên phương pháp Taguchi với một mảng trực giao $\mathrm{L}_{18}\left(3^{5}\right)$, chiều dày tấm vật liệu và tỷ số kéo là hai thông số quan trọng nhất để giảm thiểu độ chênh lệch về độ dày của sản phẩm. Kết quả cho thấy độ đồng đều tối ưu trong trường hợp đầu vào tối thiểu đã đạt được. Ở điều kiện vận hành gồm nhiệt độ $300^{\circ} \mathrm{C}$, khoảng cách $40 \mathrm{~mm}$, thời gian gia nhiệt $27 \mathrm{~s}$, tỷ số kéo 1.5 và độ dày của tấm nhựa $0.2 \mathrm{~mm}$, kết quả độ đồng đều là $0.06 \mathrm{~mm}$. Kết quả này tốt hơn kết quả nhỏ nhất trong 18 thí nghiệm trước đó là ở thí nghiệm số \#14 $(0.07 \mathrm{~mm})$.

Sự đơn giản và hiệu quả của phương pháp tối ưu hóa được sử dụng trong nghiên cứu này có thể có ích trong các công nghệ sản xuất khác nhau và các xưởng sản xuất, nơi không có nhiều thời gian để phân tích và áp dụng các hàm mục tiêu phức tạp. Những lý thuyết và tính toán về Taguchi và ANOVA nhằm để các kỹ sư hiểu bản chất của quá trình, trong thực tiễn sản xuất, toàn bộ quá trình tối ưu hóa có thể được thực hiện bởi 
Minitab ${ }^{\circledR}$, vốn có thể dễ dàng cài đặt ở các xưởng sản xuất. Từ đó, nghiên cứu này có thể góp phần lấp đầy khoảng cách giữa nghiên cứu học thuật và thực tiễn sản xuất công nghiệp ngày nay.

\section{LÒ̀I CẢM ƠN}

Trong suốt quá trình thực hiện công trình nghiên cứu và thực nghiệm, nhóm tác giả xin chân thành cảm ơn quỹ nghiên cứu khoa học trường Đại học Công Nghiệp TP.HCM, Việt Nam đã tài trợ cho nghiên cứu này thông qua dự án số 20/1.1CK01. Đồng thời, các tác giả cũng xin chân thành cảm ơn giúp đỡ từ Phó Trưởng khoa Công Nghệ Hóa Học, TS. Trần Nguyễn Minh Ân cùng cô quản lý thiết bị khoa Công Nghệ Hóa Học, trường đại học Công Nghiệp TP.HCM, Việt Nam, đã nhiệt tình cho nhóm mượn bơm hút chân không để tiến hành thực nghiệm tại phòng thí nghiệm F0.06.

\section{TÀI LIỆ THAM KHẢO}

[1] J. L. Throne, "Introduction to Thermoforming," in Understanding Thermoforming, ed: Carl Hanser Verlag GmbH \& Co. KG, 2008, pp. 1-7.

[2] T. K. Nguyen and B.-K. Lee, "Investigation of processing parameters in micro-thermoforming of microstructured polystyrene film," Journal of Mechanical Science and Technology, vol. 33, pp. 5669-5675, December 012019.

[3] P. Bourgin, I. Cormeau, and T. Saint-Matin, "A first step towards the modelling of the thermoforming of plastic sheets," Journal of Materials Processing Technology, vol. 54, pp. 1-11, 1995/10/01/ 1995.

[4] G. Sala, L. Di Landro, and D. Cassago, "A numerical and experimental approach to optimise sheet stamping technologies: polymers thermoforming," Materials \& Design, vol. 23, pp. 21-39, 2002/02/01/ 2002.

[5] C. P. J. O’Connor, G. Menary, P. J. Martin, and E. McConville, "Finite element analysis of the thermoforming of Polypropylene," International Journal of Material Forming, vol. 1, pp. 779-782, 2008/04/01 2008.

[6] C. P. J. O'Connor, P. J. Martin, and G. Menary, "Viscoelastic Material Models of Polypropylene for Thermoforming Applications," International Journal of Material Forming, vol. 3, pp. 599-602, 2010/04/01 2010.

[7] M. Ghobadnam, P. Mosaddegh, M. Rezaei Rejani, H. Amirabadi, and A. Ghaei, "Numerical and experimental analysis of HIPS sheets in thermoforming process," The International Journal of Advanced Manufacturing Technology, vol. 76, pp. 1079-1089, 2015/02/01 2015.

[8] Y.-W. Chang and J.-H. Cheng, "Numerical and experimental investigation of polycarbonate vacuum-forming process," Journal of the Chinese Institute of Engineers, vol. 36, pp. 831-841, 2013/10/01 2013.

[9] T. Azdast, A. Doniavi, S. Rash Ahmadi, and E. Amiri, "Numerical and experimental analysis of wall thickness variation of a hemispherical PMMA sheet in thermoforming process," The International Journal of Advanced Manufacturing Technology, vol. 64, pp. 113-122, 2013/01/01 2013.

[10] M. K. Warby, J. R. Whiteman, W. G. Jiang, P. Warwick, and T. Wright, "Finite element simulation of thermoforming processes for polymer sheets," Mathematics and Computers in Simulation, vol. 61, pp. 209$218,2003 / 01 / 30 / 2003$.

[11] C. Yang and S.-W. Hung, "Modeling and Optimization of a Plastic Thermoforming Process," Journal of Reinforced Plastics and Composites, vol. 23, pp. 109-121, 2004/01/01 2004.

[12] W. D. O. Leite, J. C. Campos Rubio, F. Mata Cabrera, A. Carrasco, and I. Hanafi, "Vacuum Thermoforming Process: An Approach to Modeling and Optimization Using Artificial Neural Networks," Polymers, vol. 10, p. 143, 2018. 
[13] R. McCool, P. J. Martin, and E. Harkin-Jones, "Process modelling for control of product wall thickness in thermoforming," Plastics, Rubber and Composites, vol. 35, pp. 340-347, 2006/10/01 2006.

[14] A. Makradi, S. Belouettar, S. Ahzi, and S. Puissant, "Thermoforming process of amorphous polymeric sheets: Modeling and finite element simulations," Journal of Applied Polymer Science, vol. 106, pp. 1718-1724, 2007.

[15] A. Makradi, S. Ahzi, S. Belouettar, and D. Ruch, "Thermoforming process of semicrystalline polymeric sheets: Modeling and finite element simulations," Polymer Science Series A, vol. 50, pp. 550-557, 2008/05/01 2008.

[16] H. Hosseini, B. V. Berdyshev, and A. Mehrabani-Zeinabad, "Rheological modeling of fracture in plug-assisted vacuum thermoforming," Journal of Applied Polymer Science, vol. 117, pp. 171-177, 2010/07/05 2010.

[17] F. Abbassi, I. Elfaleh, S. Mistou, A. Zghal, M. Fazzini, and T. Djilali, "Experimental and numerical investigations of a thermoplastic composite (carbon/PPS) thermoforming," Structural Control and Health Monitoring, vol. 18, pp. 769-780, 2011/11/01 2011.

[18] C. Yang and S. W. Hung, "Optimising the thermoforming process of polymeric foams: an approach by using the Taguchi method and the utility concept," The International Journal of Advanced Manufacturing Technology, vol. 24, pp. 353-360, 2004/09/01 2004.

[19] J.-C. Yu, X.-X. Chen, T.-R. Hung, and F. Thibault, "Optimization of extrusion blow molding processes using soft computing and Taguchi's method," Journal of Intelligent Manufacturing, vol. 15, pp. 625-634, 2004/10/01 2004.

[20] T. K. Nguyen, C. J. Hwang, and B.-K. Lee, "Numerical investigation of warpage in insert injection-molded lightweight hybrid products," International Journal of Precision Engineering and Manufacturing, vol. 18, pp. 187-195, 2017/02/01 2017.

[21] P. G. Karagiannidis, A. C. Stergiou, and G. P. Karayannidis, "Study of crystallinity and thermomechanical analysis of annealed poly(ethylene terephthalate) films," European Polymer Journal, vol. 44, pp. 1475-1486, 2008/05/01/2008.

[22] C. A. Seneci, M. Mor, D. Fausti, G. Petrogalli, C. Remino, and V. Zacché, "Simulation and design of an oven for PET blow molding machines," in 2012 COMSOL Conference, Milan, 2012, pp. 2855-2871.

[23] N. M. Long, Vật liệu và công nghệ chất dẻo: NXB Giáo Dục, 2013.

[24] H. Atil and Y. Unver, "A Different Approach of Experimental Design: Taguchi Method," Pakistan Journal of Biological Sciences, vol. 3, pp. 1538-1540, 2000.

[25] G. Taguchi, S. Chowdhury, and Y. Wu, "Introduction to the Signal-to-Noise Ratio," in Taguchi's Quality Engineering Handbook, ed, 2004, pp. 221-238.

[26] N. K. Triều, N. C. Thông, N. V. Trung, T. A. Tuấn, N. Q. Vinh, and T. T. Vũ, "Úng dụng lý thuyết thiết kế tiên đề vào thiết kế và chế tạo một thiết bị nhiệt định hình," Tạp chí Khoa học và công nghệ - Truờng đại hoc Công nghiệp Thành phố Hồ Chí Minh, vol. 11, 2019.

[27] G. Taguchi, S. Chowdhury, and Y. Wu, "Fundamentals of Data Analysis," Taguchi's Quality Engineering Handbook, pp. 506-514, 2004/10/22 2004.

Ngày nhận bài: 15/01/2020

Ngày chẩp nhận đăng: 09/06/2020

C 2020 Trường Đại học Công nghiệp Thành phố Hồ Chí Minh 